Discrete Comput Geom 489-505 (2003)

DOI: $10.1007 /$ s00454-003-2797-9

Geometry

\title{
Illuminating Disjoint Line Segments in the Plane*
}

\author{
Csaba D. Tóth \\ Department of Computer Science, University of California at Santa Barbara, \\ Santa Barbara, CA 93106, USA \\ toth@cs.ucsb.edu
}

\begin{abstract}
It is shown that a set of $n$ disjoint line segments in the plane can always be illuminated by $\lfloor(n+1) / 2\rfloor$ light sources, improving an earlier bound of $\lfloor 2 n / 3\rfloor$ due to Czyzowicz et al. It is also shown that $\lfloor 4(n+1) / 5\rfloor$ light sources are always sufficient and sometimes necessary to illuminate the free space and both sides of $n$ disjoint line segments for every $n \geq 2$.
\end{abstract}

\section{Introduction}

Illumination of convex sets was first studied by L. Fejes Tóth [8] who proved that $4 n-7$ light sources are always sufficient and sometimes necessary to illuminate the boundaries of $n$ disjoint compact sets in the plane for every $n \geq 3$. He also proved that $2 n-2$ light sources are always sufficient and sometimes necessary to illuminate the boundaries of $n$ disjoint disks for every $n \geq 3$. Ever since, this illumination problem has been studied for several subclasses of compact sets. Tight bounds are not known for any other cases. For $n$ disjoint homothetic triangles, there are almost matching upper and lower bounds of $n+1$ and $n$ [6]. For $n$ disjoint axis-parallel rectangles, the maximum number of necessary light sources is between $n-1$ and $n+1$ in the worst case [11], [20]. Urrutia conjectures [20] that $n+c$ light sources always suffice to monitor $n$ disjoint convex quadrilaterals or $n$ disjoint triangles in the plane, $c$ is a constant.

Given a set $D$ of objects in the plane, a set $S$ of points (light sources) collectively illuminate a point set $P$ if for every point $p \in P$ there is a light source $s \in S$ such that the open line segment $s p$ is disjoint from all objects in $D$. They illuminate the object set $D$ if they illuminate the boundary of every object in $D$. This reflects Valentine's notion of

\footnotetext{
* Work on this paper was supported by the Berlin-Zürich European Graduate Program "Combinatorics, Geometry, and Computation," financed by the German Science Foundation (DFG) and ETH Zürich.
} 
illumination [21]. (We note that Hadwiger [9] defined a different notion of illumination for convex sets with non-empty interiors. See [16] for a survey on Hadwidger's problem.)

Illumination in the presence of disjoint objects can refer to two problems which differ in their target sets: (1) illumination of the set of objects and (2) illuminating the free space of objects (that is, the complement of the union of the objects, $\mathbb{E}^{2} \backslash \bigcup D$ ). Interestingly enough, all known bounds for objects with non-empty interiors equally apply to both problems. Our two main theorems show that this is not the case for disjoint line segments in general position. The line segments are in general position if there are no three collinear segment endpoints and no two parallel segments.

Theorem 1 (Illuminating Segments). For any set $L$ of $n$ disjoint line segments in the plane in general position, there are $\lfloor(n+1) / 2\rfloor$ light sources in $\mathbb{E}^{2} \backslash \bigcup L$ that collectively illuminate $\bigcup L$.

For the illumination of $n$ line segments, the best known construction, due to Zaks [22], requires $4 n / 9-2$ light sources. Theorem 1 improves upon the best previously known upper bound, 「2n/37, which is due to Czyzowicz et al. [7]. An asymptotics of $n / 2+O(1)$ was conjectured by Czyzowicz et al. [5].

Theorem 2 (Strong Illumination of the Free Space). For any set $L$ of $n, n \geq 2$, disjoint line segments in the plane in general position, there are $\lfloor 4(n+1) / 5\rfloor$ light sources in $\mathbb{E}^{2} \backslash \bigcup L$ that collectively illuminate $\mathbb{E}^{2} \backslash \bigcup L$. This bound is tight.

If every object is a curve, then each point on the boundary of an object can be illuminated from either side of the curve: this leads to a problem of illuminating line segments from both sides, suggested by Welzl. Here every point of every given line segment needs to be illuminated by at least two light sources, one on each side of the supporting line of the segment.

Theorem 3 (Illuminating Segments from Both Sides). For any set $L$ of $n, n \geq 2$, disjoint line segments in the plane in general position, there are $\lfloor 4(n+1) / 5\rfloor$ light sources in $\mathbb{E}^{2} \backslash \bigcup L$ that collectively illuminate every point $p \in \ell, \ell \in L$, from both sides of $\ell$. This bound is tight.

Our theorems give combinatorial bounds on the worst case number of necessary light sources. Determining the minimal number of light sources for given sets of segments is NP-hard [18], like many other art gallery type optimization problems.

Other Variants of Illumination in the Presence of Line Segments. O'Rourke [17] considered the model of weak illumination: The open segment $s p$ between a light source $s$ and a target point $p$ can contain a segment of $L$, but it cannot properly cross any segment of $L$. He proved that $\lfloor 2 n / 3\rfloor$ light sources are always sufficient and sometimes necessary to illuminate the free space of $n$ disjoint segments weakly, if the light sources can be anywhere in $\mathbb{E}^{2}$ and $n>5$. Tóth [19] showed that $\lfloor 2(n+1) / 3\rfloor$ light sources in $\mathbb{E}^{2} \backslash \bigcup L$ are always sufficient and sometimes necessary to illuminate the free space weakly. 
Czyzowicz et al. [7] required that every line segment be entirely illuminated by one light source. They have shown that for that problem $\lceil 2 n / 3\rceil$ light sources in $\mathbb{E}^{2} \backslash \bigcup L$ are always sufficient and sometimes necessary.

Jennings and Lenhart [12] allowed one to place an arbitrary number of light sources on selected line segments. They proved that there are always at most $\lfloor n / 2\rfloor$ segments from $L$ such that light sources placed at those segments can illuminate all the other segments, and this bound is tight.

Proof Technique. Our allocation of light sources extends the so-called matching technique, which yields tight bounds for some illumination problems [17], [19]. Usually, the free space $\mathbb{E}^{2} \backslash \bigcup D$ of the set $D$ of disjoint objects is partitioned into convex cells. A dual graph is associated to the partition where the nodes represent convex cells, and two nodes are connected by an edge if a common light source can illuminate both cells. A small covering set (or, many times, simply a matching) of convex cells with common boundary points then provides a small set of light sources.

The key observation for our proofs is that the dual graph of a convex partitioning of disjoint segments (defined below) can be decomposed into paths of three or more nodes (such a decomposition is called a $\left\{P_{\geq 3}\right\}$-factor). Theorem 1 is based on a weaker property, namely, the dual graph has a partition into paths of two or three nodes (a $\left\{P_{1}, P_{2}\right\}$-factor).

We apply Kaneko's characterization of graphs with $\left\{P_{\geq 3}\right\}$-factors [13]. Recently, Kano et al. [14] gave a simple proof to Kaneko's theorem, and Hartvigsen and Hell [10] presented a polynomial algorithm which finds an actual $\left\{P_{\geq 3}\right\}$-factor or states that none exists. $\left\{P_{2}, P_{3}\right\}$-factors were characterized by Akiyama et al. [1]. We remark that finding a maximum $P_{3}$ packing (i.e., a set of disjoint induced paths of three nodes) in a given graph is known to be NP-hard [15] even for planar graphs [4].

Organization. We define a simple (dual) graph on a convex partitioning of the free space in Section 2. Then, in Section 3, we show that the dual graph has a $\left\{P_{\geq 3}\right\}$-factor applying Kaneko's theorem. Finally, we use a $\left\{P_{\geq 3}\right\}$-factor of the dual graph to allocate light sources and prove our Theorems 1-3 in Sections 4 and 5.

\section{Convex Partitioning and Dual Graphs}

In this section we describe a simple method to partition the free space of disjoint segments into convex pieces. Then we define two graphs on the convex partition such that the interiors of two cells represented by adjacent nodes in the graph can be illuminated by one light source.

Let $L$ be a set of $n$ disjoint line segments in the plane. Consecutively, extend every line segment $e_{1} e_{2} \in L$ beyond its endpoint $e_{1}$ (and $e_{2}$ ) until it hits the relative interior of another line segment or a previously drawn extension at point $\tau\left(e_{1}\right)$ (and $\tau\left(e_{2}\right)$ ). If the extension does not hit anything then $\tau\left(e_{i}\right)$ is a ray pointing to infinity. Starting with $n$ pairwise disjoint and non-collinear line segments, we obtain a partition of the plane into $n+1$ convex open regions [17].

The convex partitioning induced by $L$ is not unique. It depends on the order in which the segments are extended. For our purposes, any partition is just fine. (It was shown in 


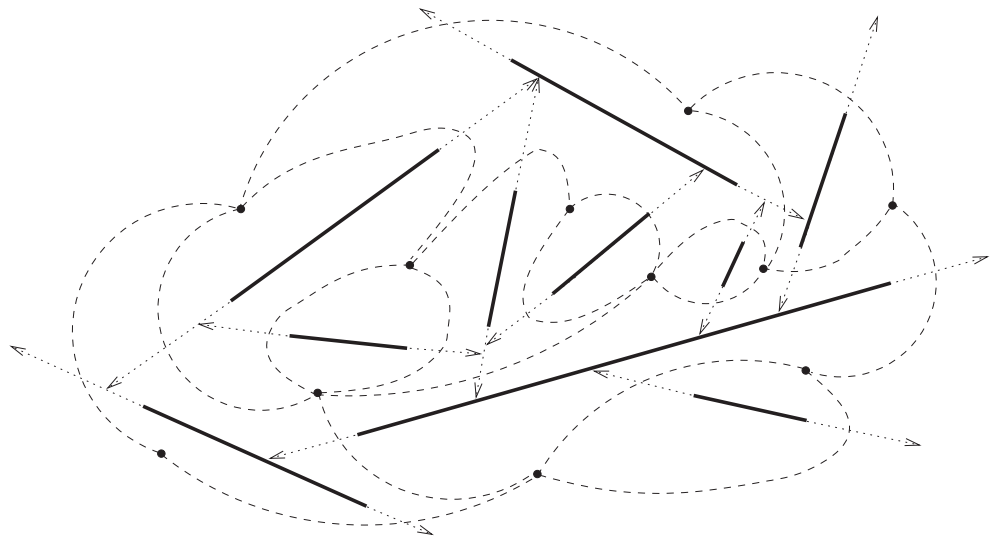

Fig. 1. A convex partitioning and the corresponding planar graph $H$.

[19] that the line segments can be extended while keeping them disjoint such that the partitioning algorithm run on the extended segments returns a unique partition.) If the partitioning algorithm extends a segment $\ell=e_{1} e_{2}$ beyond $e_{1}$ to a (possibly previously extended) segment, then we call the point $\tau\left(e_{1}\right)$ a $T$-junction. If it extends $e_{1} e_{2}$ beyond $e_{1}$ to infinity, then we call the ray $\tau\left(e_{1}\right)$ an I-junction.

We define the graph $H$ of the convex partition as follows: The node set $V(H)$ corresponds to the set of convex cells. To either endpoint of every segment $e_{1} e_{2} \in L$, we associate an edge in $H$ : the edge associated to $e_{i}, i=1,2$, connects the two nodes corresponding to the two cells which lie along $e_{i} \tau\left(e_{i}\right)$ on opposite sides and which are incident to $\tau\left(e_{i}\right)$. (See Fig. 1.) Since every segment gives rise to exactly two edges, the graph $H$ has exactly $2 n$ edges. Notice that $H$ may have double edges if the extension $\tau\left(e_{1} e_{2}\right)$ of a line segment $e_{1} e_{2} \in L$ lies completely in the common boundary of two cells. Observe that $H$ is a planar graph without loops.

We obtain a simple graph $K$ from $H$ by replacing every double edge by a single edge. For a node $v \in V(H)=V(K)$, we denote by $R_{v}$ the closure of the cell corresponding to $v$. Similarly, for a set of nodes $S \subseteq V(K)$, let $R_{S}=\bigcup\left\{R_{v}: v \in S\right\}$.

\section{The Graph $K$ Has a $\left\{P_{\geq 3}\right\}$-Factor}

We write $P_{i}$ as a shorthand for a path consisting of $i, i \in \mathbb{N}$, nodes. A partition of a simple graph $G$ into $P_{3}$ 's, $P_{4}$ 's, and $P_{5}$ 's is a $\left\{P_{3}, P_{4}, P_{5}\right\}$-factor of $G$. Every path of more than five nodes has a $\left\{P_{3}, P_{4}, P_{5}\right\}$-factor, this justifies the shorthand notation $\left\{P_{\geq 3}\right\}$-factor for $\left\{P_{3}, P_{4}, P_{5}\right\}$-factors. We can now formulate the main lemma of this paper.

Lemma 4. The graph $K$ has $a\left\{P_{\geq 3}\right\}$-factor if $|V(K)| \geq 3$.

In our illumination problems, it will suffice to apply the following two immediate corollaries. 
Corollary 5. $K$ has a $P_{3}$-packing which covers at least $3\lceil|V(K)| / 5\rceil$ nodes if $|V(K)|$ $\geq 3$.

Corollary 6. $K$ has a $\left\{P_{2}, P_{3}\right\}$-factor if $|V(K)| \geq 1$.

\subsection{Kaneko's Theorem}

The main tool for the proof of Lemma 4 is Kaneko's theorem [13] which characterizes $\left\{P_{\geq 3}\right\}$-factors.

Theorem 7 (Kaneko's Theorem). A simple graph $G$ has a $\left\{P_{\geq 3}\right\}$-factor if and only if

$$
\operatorname{sun}(G-S) \leq 2|S|, \quad \text { for every } S \subseteq V(G) .
$$

The term $\operatorname{sun}(G-S)$ requires further explanation.

Definition 8. A graph $F$ is factor-critical if for every $v \in V(F), F-\{v\}$ has a perfect matching (1-factor).

A single node is a sun graph. All other sun graphs can be obtained from a factorcritical graph $F$ with $V(F)=\left\{v_{1}, v_{2}, \ldots, v_{k}\right\}$, by adding new vertices $u_{1}, u_{2}, \ldots, u_{k}$ and new edges $v_{1} u_{1}, v_{2} u_{2}, \ldots, v_{k} u_{k}$. (Figure 2 depicts four suns.)

Let $\operatorname{sun}(G)$ denote the number of connected sun components of a simple graph $G$.

A factor-critical graph with $k$ nodes has at least $k$ edges if $k>1$. To see this, observe that every factor-critical graph is connected, and it has no leaves. Note that a single node is also factor-critical and the corresponding sun graph is a path $P_{2}$. Consequently, a sun with $2 k$ nodes, $k>1$, has at least $2 k$ edges.

\subsection{Proof of Lemma 4}

Before we proceed with the proof of Lemma 4, we need some definitions. The wheel subgraphs (defined below) will have special role if they form the factor-critical core of a sun subgraph of $K$.

Definition 9. For a graph $G$ and $S \subseteq V(G)$, denote the degree of $S$ in $G$ by $\operatorname{deg}_{G}(S)$ (i.e., $\operatorname{deg}_{G}(S)$ is the number of edges connecting nodes of $S$ to nodes of $V(G) \backslash S$ ).

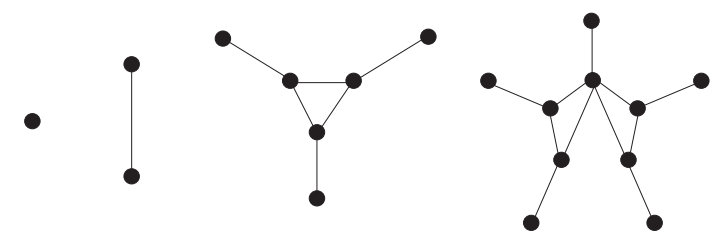

Fig. 2. Four sun graphs. 


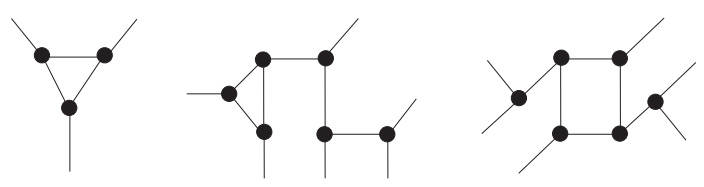

Fig. 3. Three wheels with all their outgoing edges.

Let $G(S)$ be the subgraph of $G$ induced by $S$, and let $G-S$ be the subgraph induced by $V(G) \backslash S$. Denote by $E_{G}(S)=E(G(S))$ the set of edges induced by $S$ in $G$.

Definition 10. A vertex set $S \subset V(G)$ of a graph $G$ is a wheel in $G$, if $S$ induces a connected graph, $\left|E_{G}(S)\right|=|S|, \operatorname{deg}_{G}(S)=|S|$, and $\operatorname{deg}_{G}(v)>2$ for every $v \in S$ (Fig. 3).

The key step to the application of Kaneko's theorem is the following lemma. The rather technical proof is postponed to the next subsection.

Lemma 11. Let $W$ be a proper subset of $V(K)$. Then

$$
\left|E_{K}(W)\right| \leq 2|W|-p(W)-r(W),
$$

where $p(W)$ is the number of nodes in $W$ whose degree is 2 in both $K$ and $K(W)$, and $r(W)$ is the maximum number of disjoint subsets of $W$ which are wheels in both $K$ and $K(W)$.

Now we are ready to establish our main lemma and show that $K$ admits a $\left\{P_{\geq 3}\right\}$-factor.

Proof of Lemma 4. Suppose that inequality (1) does not hold for the graph $K$ of the convex partitioning. Choose a subset of nodes $S \subset V(K)$, where $\operatorname{sun}(K-S)-2|S|$ is maximal.

If $S=\emptyset$, then $K-S=K$. $K$ has only one connected component, and does not have any leaves if $|V(K)|>2$. Therefore, $\operatorname{sun}(K)>0$ implies $|V(K)| \leq 2$.

Now assume that $S$ is non-empty. Delete from $K$ all components of $K-S$ which are not sun and denote the resulting graph by $K^{\prime}$ (Fig. 4). We wish to apply Lemma 11 and, in particular, inequality (2) with $W=V\left(K^{\prime}\right)$. For this reason, we introduce parameters for the number of certain types of sun components of $K-S$. The $P_{1}$ 's, $P_{2}$ 's, and all other suns are considered separately.

Let $c_{2}$ and $c_{3}$ be the number of single node components $v \in V(K) \backslash S$ with $\operatorname{deg}_{K}(v)=$ 2 and $\operatorname{deg}_{K}(v) \geq 3$, respectively. (Note that here $\operatorname{deg}_{K^{\prime}}(v)=\operatorname{deg}_{K}(v)$.)

Let $d_{2}, d_{3}$, and $d_{4}$ be the number of $P_{2}$ components $P \subset V(K) \backslash S$ such that $\operatorname{deg}_{K}(P)=$ $2, \operatorname{deg}_{K}(P)=3$, and $\operatorname{deg}_{K}(P) \geq 4$, respectively. Observe that $\operatorname{deg}_{K}(P)=2$ (resp., $\operatorname{deg}_{K}(P)=3$ ) means that $P$ contains two (resp., one) nodes with $\operatorname{deg}_{K}(v)=2$.

Consider the sun components of $K-S$ with more than two nodes. Let the total number of their nodes be $2 k$. They have $k$ leaves. Let $f_{2}$ and $f_{3}$ be the total number of their leaves $v$ with $\operatorname{deg}_{K}(v)=2$ and $\operatorname{deg}_{K}(v)>2$, respectively. Let $r_{1}$ and $r_{2}$ be the number sun components of $K-S$ with more than two nodes, whose factor-critical subgraph is wheel 


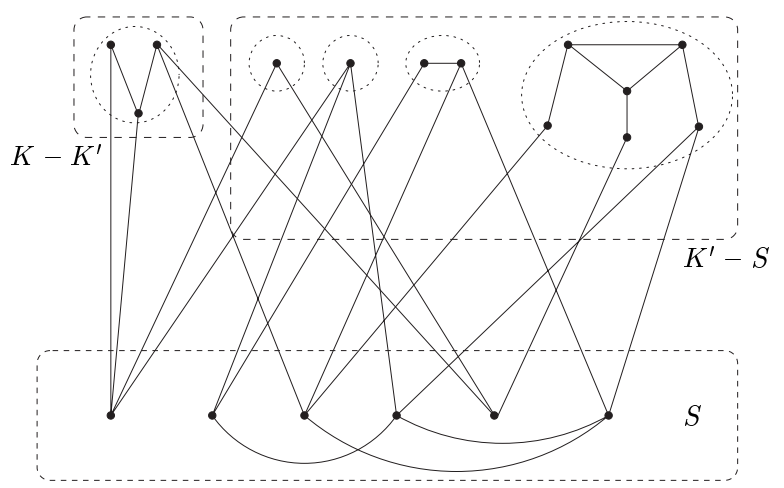

Fig. 4. Illustration for the proof of Lemma 4.

and non-wheel, respectively. These components have at least $2 k+r_{2}$ interior edges and at least $2 k-f_{2}$ edges connect them to $S$.

Using these parameters, we can give a lower bound to the number of edges in $K^{\prime}$ by $\left|E\left(K^{\prime}\right)\right| \geq \operatorname{deg}_{K^{\prime}}(S)+\left(d_{2}+d_{3}+d_{4}\right)+\left(2 k+r_{2}\right)$ where $\operatorname{deg}_{K^{\prime}}(S) \geq 2 c_{2}+3 c_{3}+2 d_{2}+$ $3 d_{3}+4 d_{4}+\left(2 k-f_{2}\right)$. The number of nodes having degree 2 in both $K^{\prime}$ and $K$ is at least $c_{2}+2 d_{2}+d_{3}+f_{2}$.

Inequality (2) with $W=V\left(K^{\prime}\right)$ is written as

$$
\begin{gathered}
2 c_{2}+3 c_{3}+3 d_{2}+4 d_{3}+5 d_{4}+4 k+r_{2}-f_{2} \\
\leq 2\left(|S|+c_{2}+c_{3}+2 d_{2}+2 d_{3}+2 d_{4}+2 k\right)-\left(c_{2}+2 d_{2}+d_{3}+f_{2}\right)-r_{1}, \\
c_{2}+c_{3}+d_{2}+d_{3}+d_{4}+r_{1}+r_{2} \leq 2|S| .
\end{gathered}
$$

The number of sun components is at most $2|S|$. According to Kaneko's theorem, $K$ has a $\left\{P_{\geq 3}\right\}$-factor.

\subsection{The Structure of Graphs $H$ and $K$}

Recall that $K$ is the graph obtained from $H$ by replacing double edges by simple edges, furthermore, $|V(K)|=n+1$ and $|E(K)| \leq|E(H)|=2 n$. If $H$ has $d$ double edges, then $|E(K)| \leq 2 n-d=2|V(K)|-2-d$.

Denote by $q$ the number of line segments which are extended to a complete line by the convex partitioning algorithm. Assuming general position, $q \in\{0,1\}$. Denote this line by $\ell_{0} \in L$ if it exists. Observe that there is no cutting edge in $H$ or in $K$ if $n>1$.

Lemma 12. The number of 2-edge cuts in $H$ is $q$.

Proof. Consider a proper subset $S \subset V(H)$. The region $R_{S}$ is connected if $S$ induces a connected subgraph. The boundary of $R_{S}$ is composed of pieces of the extended line 
segments of $L$. We distinguish two cases:

Case 1: $R_{S}$ or $\mathbb{E}^{2} \backslash R_{S}$ is a bounded region. Every bounded polygonal domain has at least three vertices. Every vertex corresponds to a T-junction. Every T-junction on the boundary of $R_{S}$, in turn, corresponds to an edge of $H$ in $\operatorname{deg}_{H}(S)$, hence $\operatorname{deg}_{H}(S) \geq 3$.

Case 2: Both $R_{S}$ and $\mathbb{E}^{2} \backslash R_{S}$ are unbounded regions. There are at least two I-junctions on the boundary of $R_{S}$. If there are at least two line segments on the boundary of $R_{S}$, then there is also at least one T-junction on the boundary (there are no collinear line segments) and therefore, $\operatorname{deg}_{H}(S) \geq 3$.

A set $S \subset V(H)$ for which both $R_{S}$ and $\mathbb{E}^{2} \backslash R_{S}$ are unbounded can have $\operatorname{deg}_{H}(S)=2$ if and only if there is exactly one line segment on the boundary of $R_{S}$. In this case the line segment $\ell$ on the boundary of $R_{S}$ is extended to infinity in both directions, and $\ell=\ell_{0}$.

Corollary 13. For every node $v$ with $\operatorname{deg}_{H}(v)=2, R_{v}$ is a half-plane of $\ell_{0}$.

Corollary 14. If $S \subseteq V(G)$ such that $\operatorname{deg}_{H}(S)=2$, then $R_{S}$ is a half-plane of $\ell_{0}$.

Lemma 15. Let $S$ be a proper subset of $V(H)$ such that $S$ induces a connected subgraph. Then

$$
2|S| \leq\left|E_{H}(S)\right|+\operatorname{deg}_{H}(S) .
$$

Proof. Let $x$ be the number of the line segments $e_{1} e_{2} \in L$ such that relint $\left(\tau\left(e_{1}\right) \tau\left(e_{2}\right)\right) \subset$ $\operatorname{int}\left(R_{S}\right)$. Note that either endpoint of a segment $e_{1} e_{2} \subset \operatorname{int}\left(R_{S}\right)$ corresponds to an edge in $E_{H}(S)$. Let $y$ be the number of segment endpoints which corresponds to an edge in $E_{H}(S)$ but whose segment relint $\left(\tau\left(e_{1}\right) \tau\left(e_{2}\right)\right)$ is not fully in $\operatorname{int}\left(R_{S}\right)$. Finally, let $z$ be the number of segments on the boundary of $R_{S}$ completely disjoint from int $\left(R_{S}\right)$. Using these parameters, we estimate $|S|,\left|E_{H}(S)\right|$, and $\operatorname{deg}_{H}(S)$.

For estimating $|S|$, we keep track of how $R_{S}$ is partitioned by our convex partitioning algorithm when the segment extensions $e_{1} \tau\left(e_{1}\right), e_{2} \tau\left(e_{2}\right), \ldots, e_{2 n} \tau\left(e_{2 n}\right)$ are added oneby-one. Let $R(0)=R_{S} \backslash \bigcup L$. Denote by $h$ the number of holes of $R(0)$. In particular, every segment of $L$ fully in $\operatorname{int}\left(R_{S}\right)$ forms a hole, hence $h \geq x$. Moreover if $\mathbb{E}^{2} \backslash R_{S}$ is bounded, then $h \geq x+1$. Put $R_{S}(j):=R_{S} \backslash \bigcup_{i=1}^{j} e_{i} \tau\left(e_{i}\right)$, for $j=1,2, \ldots, 2 n$. By deleting one more segment $e_{i} \tau\left(e_{i}\right)$ intersecting $\operatorname{int}\left(R_{S}\right)$, either the number of connected components of $R_{S}(j+1)$ increases by one, or the the total number of holes in all components of $R_{S}(j+1)$ drops by one. As $R_{S}(2 n)$ has no holes and at least $2 x+y$ extensions intersect the interior of $R_{S}$, there must be $2 x+y-h$ steps increasing the number of connected components by one each.

$\left|E_{H}(S)\right|=2 x+y$ follows from the definition of parameters $x$ and $y$. For estimating $\operatorname{deg}_{H}(S)$, consider the consecutive line segments along every connected component of the boundary of $R_{S}$. Every pair of consecutive line segments on the boundary forms a T-junction and corresponds to an edge in $\operatorname{deg}_{H}(S)$. The extensions of the first and the last segment endpoints on the boundary of an unbounded component correspond to two I-junctions. This implies that $\operatorname{deg}_{H}(S) \geq z+y$ if $R_{S}$ or $\mathbb{E}^{2} \backslash R_{S}$ is bounded, and $\operatorname{deg}_{H}(S) \geq z+y+1$ if both are unbounded. 
We can summarize the cases as follows. If the complement $\mathbb{E}^{2} \backslash R_{S}$ is bounded, then $|S| \leq x+y$ and $\left|E_{H}(S)\right|+\operatorname{deg}_{H}(S) \geq 2 x+2 y+z$, so our proof is complete. Otherwise we have $|S| \leq x+y+1$. If $R_{S}$ is bounded, then the convex hull $\operatorname{conv}\left(R_{S}\right)$ has at least three sides. On every curve on the boundary of $R_{S}$ between two endpoints of a side of $\operatorname{conv}\left(R_{S}\right)$, there is a segment on the boundary of $R_{S} \operatorname{disjoint} f$ from $\operatorname{int}\left(R_{S}\right)$, hence $z \geq 3$. This implies that $\left|E_{H}(S)\right|+\operatorname{deg}_{H}(S) \geq 2 x+2 y+3$. If both $R_{S}$ and $\mathbb{E}^{2} \backslash R_{S}$ are unbounded, then $\left|E_{H}(S)\right|+\operatorname{deg}_{H}(S) \geq 2 x+2 y+z+1$ and $z \geq 1$ since $\operatorname{conv}\left(R_{S}\right)$ has at least one side.

Corollary 16. If $S \subset V(K)$ is a wheel in $K$ and $\left|E_{H}(S)\right|=|S|$, then either $\mathbb{E}^{2} \backslash R_{S}$ is bounded or $R_{S}$ is a half-plane of $\ell_{0}$.

Proof. Suppose that $S$ is a wheel with $k$ nodes and $S$ induces at most $k$ edges in $H$. Clearly, $S \neq V(H)$. We have

$$
2 k=\left|E_{H}(S)\right|+\operatorname{deg}_{K}(S) \leq\left|E_{H}(S)\right|+\operatorname{deg}_{H}(S) \leq k+|S|=2 k .
$$

That is, inequality (3) holds with equality. It follows from the argument of the previous proof that either $\mathbb{E}^{2} \backslash R_{S}$ is bounded or both $R_{S}$ and $\mathbb{E}^{2} \backslash R_{S}$ are unbounded and $z=1$.

Proof of Lemma 11. We know that $|E(H)|=2|V(H)|-2$. Applying Lemma 15 to every component of $H-W$, we have $\left|E_{H}(W)\right| \leq 2|W|-2$.

It suffices to show that $W$ induces at least $p(W)+r(W)-2$ double edges in $H$. Note that the nodes of the wheels do not have degree 2 in $K$. According to Corollary 16, every wheel $S \subset W$ induces a double edge of $H$ unless $R_{S}$ is a half-plane of $\ell_{0}$ or $\mathbb{E}^{2} \backslash R_{S}$ is bounded. According to Corollary 13, every node $v \in W$ with $\operatorname{deg}_{K}(v)=2$ is incident to a double edge of $H$ unless $R_{v}$ is a half-plane of $\ell_{0}$. It is possible, however, that two nodes $v \in W$ and $w \in W$ with $\operatorname{deg}(v)=\operatorname{deg}(w)=2$ are incident to the same double edge of $H$. Consider a path $P \subseteq W$ with $\operatorname{deg}_{K}(v)=2$ and $\operatorname{deg}_{H}(v)>2$ for every $v \in P$, and note that $\operatorname{deg}_{K}\left(P^{\prime}\right)=2$ for every subpath $P^{\prime} \subseteq P$. Therefore, the nodes of $P$ are incident to $|P|$ double edges of $H$ unless $R_{P}$ contains a half-plane of $\ell_{0}$. Since $\ell_{0}$ have only two half-planes, and neither is disjoint from a region $R_{S}$ whose complement is bounded, we concluded that $H(W)$ must have at least $p(W)+r(W)-2$ double edges.

\section{Illumination of the Free Space and Illuminating Both Sides of Segments}

\section{1. $P_{3}$-Packings of the Graph $K$}

First we introduce the notion of bad $P_{3}$ to highlight a few exceptional cases. Then we proceed with Lemma 17 which states that two light sources can basically illuminate $R_{a}$, $R_{b}$, and $R_{c}$ whenever $(a, b, c)$ forms a $P_{3}$ in $K$.

Fix a convex partition of a set $L$ of disjoint line segments. Let $e=a b$ be an edge of $H$ between the nodes $a$ and $b$. Assume that $e$ corresponds to a T-junction $\tau(e)$. We associate a small region $r_{\varepsilon}(a, e)$ to the directed edge $e(a, b)$, where $\varepsilon$ is a small positive constant. Let $r_{\varepsilon}(a, e)$ be the intersection of the closed cell $R_{a}$ and the closed disk centered at $\tau(e)$ of radius $\varepsilon$. 
Consider a $P_{3}(a, b, c)$ of $K$. Assume that the lines through the line segments $e_{1} e_{3} \in L$ and $e_{2} e_{4} \in L$ form the common boundary of $R_{a}, R_{b}$ and $R_{b}, R_{c}$, respectively, such that the segment endpoints $e_{1}$ and $e_{2}$ correspond to edges $a b$ and $b c$, respectively. We say that $(a, b, c)$ is a bad $P_{3}$ if $\tau\left(e_{3}\right) \in \tau\left(e_{2}\right) \tau\left(e_{4}\right)$ or symmetrically if $\tau\left(e_{4}\right) \in \tau\left(e_{1}\right) \tau\left(e_{3}\right)$. The region $r_{\varepsilon}\left(b, e_{3}\right)$ or $r_{\varepsilon}\left(b, e_{4}\right)$, respectively, is called the bad $\varepsilon$-corner of the bad $P_{3}$ (see Fig. 6).

Lemma 17. There is a positive constant $\varepsilon_{0}=\varepsilon_{0}(L)$ such that for all $\varepsilon, 0<\varepsilon<\varepsilon_{0}$, and every $P_{3}(a, b, c)$ of $K$, we can place two light sources in $\operatorname{int}\left(R_{a}\right) \cup \operatorname{int}\left(R_{b}\right) \cup \operatorname{int}\left(R_{c}\right)$ with the following properties:

(i) They illuminate all portions of $\bigcup L$ on the boundaries of $R_{a}, R_{b}$, and $R_{c}$.

(ii) If $(a, b, c)$ is not bad, then they illuminate all portions of $\bigcup L$ on the boundaries of $R_{a}, R_{b}$, and $R_{c}$ from the side of $R_{a}, R_{b}$, and $R_{c}$ (from two sides on the common boundaries); and they illuminate $R_{a b c} \backslash \bigcup L$.

(iii) If $(a, b, c)$ is bad, then they illuminate all portions of $\bigcup L$ on the boundaries of $R_{a}, R_{b}$, and $R_{c}$ from the side of $R_{a}, R_{b}$, and $R_{c}$ (from two sides on the common boundaries), except for the portions in the bad $\varepsilon$-corner from the side of $R_{b}$; and they illuminate $R_{a b c} \backslash \bigcup L$ except for the bad $\varepsilon$-corner.

(iv) They illuminate every $r_{2 \varepsilon}(d, e)$ where $d \in V(H) \backslash\{a, b, c\}$ and e corresponds to an edge of $H$ between $d$ and $(a, b, c)$.

Proof. Let $e_{1}$ and $e_{2}$ denote the segment endpoints which correspond to edges $a b$ and $b c$, respectively. If $\tau\left(e_{1}\right)$ (resp., $\left.\tau\left(e_{2}\right)\right)$ is a T-junction, then let $w_{1}=\tau\left(e_{1}\right)\left(w_{2}=\tau\left(e_{2}\right)\right)$. If $\tau\left(e_{1}\right)$ (resp., $\left.\tau\left(e_{2}\right)\right)$ is an I-junction, then let $w_{1}\left(w_{2}\right)$ be the intersection point of the common boundary of $R_{a}$ and $R_{b}\left(R_{b}\right.$ and $\left.R_{c}\right)$ and a bounding box of $\bigcup L$.

We place two light sources $s_{1}$ and $s_{2}$ into $\operatorname{int}\left(R_{a}\right) \cup \operatorname{int}\left(R_{b}\right) \cup \operatorname{int}\left(R_{c}\right)$ in the $\delta$ neighborhood of $w_{1}$ and $w_{2}$, where $\delta>0$ is a sufficiently small positive constant depending on $L$ and $\varepsilon$. If $e_{1} e_{2}$ is not a segment of $L$, then we place the two light sources in the interior of $R_{a}$ and $R_{b}$ (Fig. 5, left). If $e_{1} e_{2} \in L$, then we place them on opposite sides of the line through $e_{1} e_{2}$, say, into the interior of $R_{a}$ and $R_{b}$ (Fig. 5, right).

Each light source illuminates the cell in which it is lying and the boundary of this cell. If $e_{1} e_{2} \in L$, then the light source $s_{2}$ illuminates the cell $R_{c}$, except for the cone
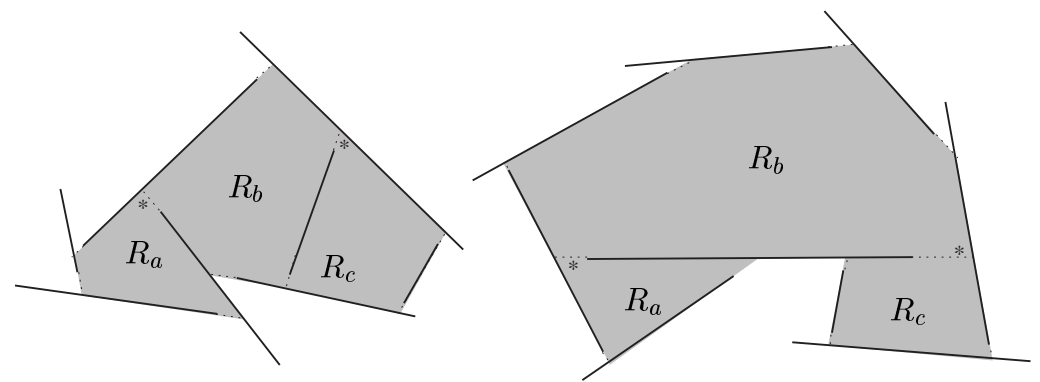

Fig. 5. Two examples where the two light sources are located in $R_{a} \cup R_{b} \cup R_{c}$ if $(a, b, c)$ is not a bad $P_{3}$. 


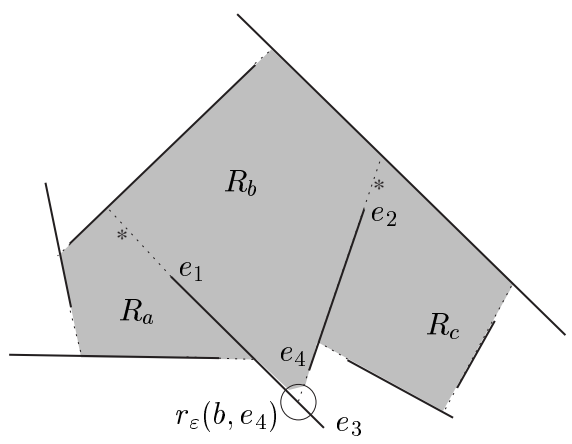

Fig. 6. Two light source do not illuminate a bad $\varepsilon$-corner of a bad $P_{3}$.

between the rays $\overrightarrow{e_{1} e_{2}}$ and $\overrightarrow{s_{2} e_{2}}$ and the portion of $e_{1} e_{2}$ on the boundary of $R_{c}$. Both are illuminated by $s_{1}$ if $\delta$ is sufficiently small.

If $e_{1} e_{2} \notin L$, then let $e_{1} e_{3} \in L$ and $e_{2} e_{4} \in L$. The light source $s_{1} \in \operatorname{int}\left(R_{a}\right)$ also illuminates cell $R_{b}$; except for the cone between the rays $\overrightarrow{e_{1} e_{3}}$ and $\overrightarrow{s_{1} e_{1}}$ and the portion of $e_{1} e_{3}$ on the boundary of $R_{b}$ from side $R_{b}$. The light source $s_{2} \in \operatorname{int}\left(R_{c}\right)$ also illuminates cell $R_{b}$; except for the cone between the rays $\overrightarrow{e_{2} e_{4}}$ and $\overrightarrow{s_{2} e_{2}}$, and the portion of $e_{2} e_{4}$ on the boundary of $R_{b}$ from side $R_{b}$. They together illuminate $R_{b}$ and its boundary except if $(a, b, c)$ is bad. In that case the intersection of the non-illuminated pieces are all in the bad corner if $\delta$ is sufficiently small with respect to $\varepsilon$.

Finally $s_{1}$ and $s_{2}$ illuminate the regions $r_{2 \varepsilon}(D, V)$ in adjacent cells if $\varepsilon$ is sufficiently small. We note only one particular case: if $(a, b, c)$ is a bad $P_{3}$, then the corner $r_{2 \varepsilon}\left(d, e_{3}\right)$ (resp., $\left.r_{2 \varepsilon}\left(d, e_{4}\right)\right)$ of the neighboring cell opposite to the bad $\varepsilon$-corner of $(a, b, c)$ is also illuminated by the light source $s_{1}$ (resp., $s_{2}$ ) (Fig. 6). Note that the upper bound on $\varepsilon$ depends only on $L$ and not on $\delta$.

For the sake of completeness, we make the following observation about the bad corners.

Proposition 18. There is a positive constant $\varepsilon_{1}=\varepsilon_{0}(L)$ such that for all $\varepsilon, 0<\varepsilon<\varepsilon_{1}$, and every $a \in V(K)$, we can place a light source in $\operatorname{int}\left(R_{a}\right)$ with the following properties:

(i) It illuminates $R_{a}$; and it illuminates all portions of $\bigcup L$ on the boundary of $R_{a}$.

(ii) It illuminate every $r_{2 \varepsilon}(d, e(a, d))$ where $v \in V(H) \backslash\{a\}$ and $e(a, d) \in E(H)$.

\subsection{Proof of Theorems 2 and 3}

(Sufficiency) According to Corollary 5, $K$ contains a $P_{3}$-packing which covers at least $3\lceil(n+1) / 5\rceil$ nodes. Place two light sources for every $P_{3}$ as described in the proof of Lemma 17 and one light source in the interior of every cell not covered by a $P_{3}$ as described in Proposition 18. The two light sources in a $P_{3}$ illuminate the interior and the boundaries of the three cells except for a bad corner in the case of bad $P_{3}$ 's. The bad corners are illuminated from the opposite side: On the other side there is either another 


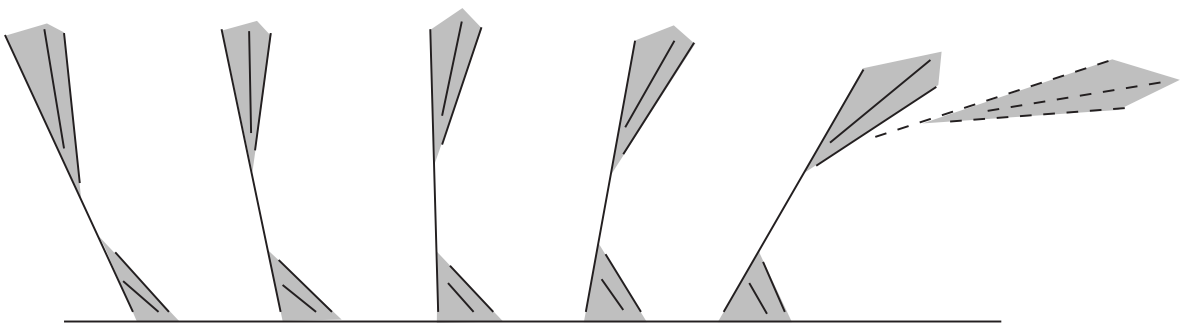

Fig. 7. A construction of $n=26$ line segments requiring 21 light sources.

$P_{3}$ of our $P_{3}$-packing and the bad corner is illuminated by Lemma 17(iv), or there is a single cell in our $P_{3}$-packing and the bad corner is illuminated by Proposition 18(ii). The total number of light sources is

$$
2\left\lceil\frac{n+1}{5}\right\rceil+\left((n+1)-3\left\lceil\frac{n+1}{5}\right\rceil\right)=\left\lfloor 4 \cdot \frac{n+1}{5}\right\rfloor .
$$

(Necessity) Figure 7 depicts a construction of $5 k+1$ line segments requiring $4 k+$ $1=\lfloor 4(n+1) / 5\rfloor$ light sources. Observe that each line segment in the interior of a shaded region requires two distinct light sources. (The maximum $P_{3}$-packing of the corresponding unique graph $K$ (Fig. 8) covers $3(k+1)$ nodes out of the total of $|V(K)|=$ $5 k+2$.

We obtain a construction on $n=5 k+2,5 k+3$, or $5 k+4$ line segments by adding one, two, or three segments shown as dashed lines in Fig. 7.

Theorem 3 says that the light source placement based on a maximum $P_{3}$-packing of $K$ is best possible in the worst case. Note, however, that there is no one-to-one correspondence between maximum $P_{3}$-packings of $K$ and optimal solutions to this illumination problem.

\section{Illuminating Segments}

\subsection{First Approach}

We wish to find a set of light sources in the free space that collectively illuminate every point of the line segments (from one side at least). We show first how an upper bound of $\lfloor 2(n+1) / 3\rfloor$ can be deduced from Corollary 6 (i.e., a maximum matching of $G$ covers at least $2\lceil(n+1) / 3\rceil$ nodes $)$.

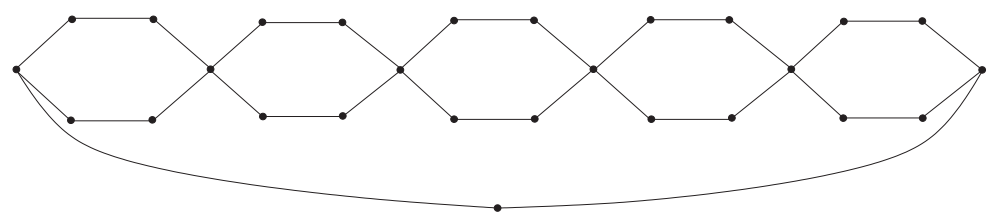

Fig. 8. The corresponding graph $K$. 
Let $a b$ be a $P_{2}$ in $K$. We say that $a b$ is a $b a d P_{2}$ if two edges of $H$ connects $a$ and $b$ (i.e., $a b$ is a double edge in $H$ ). Let $e_{1} e_{2}$ be the segment whose two endpoints correspond to the two edges of $H$ between $a$ and $b$. We call bad points of the bad $P_{2}$ the two points $\tau\left(e_{1}\right)$ and $\tau\left(e_{2}\right)$.

Lemma 19. There is a positive constant $\varepsilon_{0}=\varepsilon_{0}(L)$ such that for all $\varepsilon, 0<\varepsilon<\varepsilon_{0}$, and every $P_{2}$ of $K$, we can place a light source in $\operatorname{int}\left(R_{a}\right) \cup \operatorname{int}\left(R_{b}\right)$ with the following properties.

(i) If $a b$ is not bad, then it illuminates all portions of $\bigcup L$ on the boundaries of $R_{a}$ and $R_{b}$.

(ii) If $a b$ is bad, then it illuminates all portions of $\bigcup L$ on the boundaries of $R_{a}$ and $R_{b}$; except for parts of one of the following four disjoint intervals on the boundary of $R_{a b}$ : each interval has length $\varepsilon$ and they lie in an $\varepsilon$-neighborhood of the two bad points of ab but do not include the bad points.

Proof. Assume that the lines through $e_{1} e_{2} \in L$ form the common boundary of $R_{a}$ and $R_{b}$ such that the segment endpoint $e_{1}$ corresponds to an edge $a b \in E(H)$. If $e_{1}$ is a T-junction, then let $w_{1}=\tau\left(e_{1}\right)$; if $e_{1}$ is an I-junction, then let $w_{1}$ be the intersection of $e_{1} \tau\left(e_{1}\right)$ and a bounding box of $\bigcup L$.

If $a b$ is not bad, then we place a light source $s$ into $\operatorname{int}\left(R_{a}\right) \cup \operatorname{int}\left(R_{b}\right)$ in the $\delta$ neighborhood of $w_{1}$, where $\delta>0$ is a sufficiently small positive constant. If $s \in \operatorname{int}\left(R_{a}\right)$ $\left(s \in \operatorname{int}\left(R_{b}\right)\right)$, then $s$ illuminates $R_{a}\left(R_{b}\right)$ and it also illuminates the boundary of the other cell, except for the interior of the cone $C_{b}\left(C_{a}\right)$ between the rays $\overrightarrow{e_{1} e_{2}}$ and $\overrightarrow{s e_{1}}$. The interior of either $C_{a}$ or $C_{b}$ contains no line segments on the boundary $\partial R_{a b}$ (Fig. 9, left).

If $a b$ is a bad $P_{2}$, then the segment endpoint $e_{2}$, too, corresponds to an edge of $H$ between $a$ and $b$. We place a light source in the interior of $R_{a}$ or $R_{b}$, in the $\delta$-neighborhood of $w_{1}$ or $w_{2}$. Thus we have four disjoint regions for locating a light source $s$. In all four cases, the only portion of the boundaries $\partial R_{a} \cup \partial R_{b}$ non-illuminated by $s$ is the projection of $e_{1} e_{2}$ to the boundary $\partial R_{a b}$ from center $s$. The non-illuminated portion of the boundary can be in four disjoint intervals around the two bad points $\tau\left(e_{1}\right)$ or $\tau\left(e_{2}\right)$ depending on the four possible location of $s$. This portion can be smaller than $\varepsilon$, if $\delta$ is sufficiently small with respect to $\varepsilon$ (Fig. 9, right).
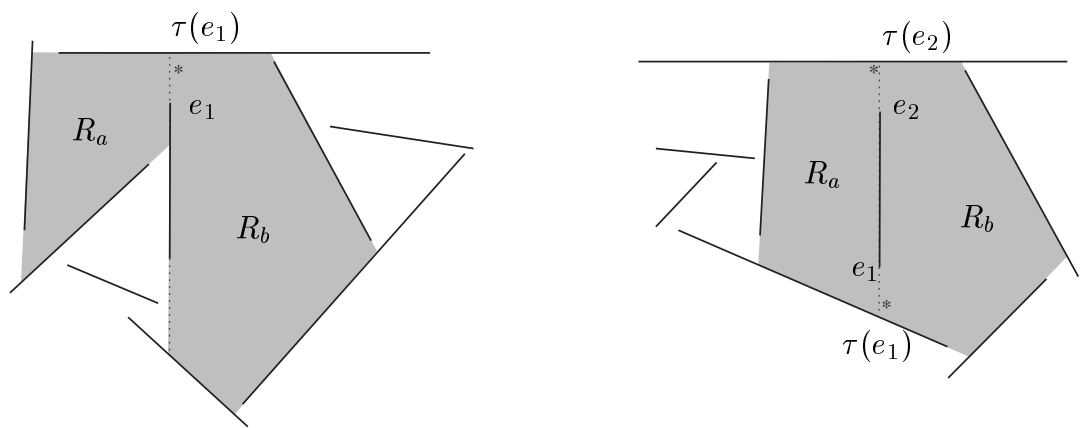

Fig. 9. A light source next to $v_{1}$ or $v_{2}$ cannot always illuminate the entire boundary of $R_{a b}$. 
Theorem 20. For any set $L$ of $n$ disjoint line segments in the plane in general position, there are $\lfloor 2(n+1) / 3\rfloor$ light sources in $\mathbb{E}^{2} \backslash \bigcup L$ that collectively illuminate $\bigcup L$.

Proof. Let $M$ be a maximum matching of $K$. Place one light source into every pair of $M$ as described in Lemma 19 , and one light source in the interior of every cell not covered by $M$. By Corollary 6 , we used no more than $\lceil(n+1) / 3\rceil+(n+1-2\lceil(n+1) / 3\rceil)=$ $\lfloor 2(n+1) / 3\rfloor$ light sources. For every bad pair $a b \in M$, there is a short interval which is not illuminated by the light source assigned to $a b$. All non-illuminated small intervals can be illuminated from the other side by choosing $\varepsilon$ sufficiently small in Lemma 19 .

\subsection{Proof of Theorem 1}

Let $F$ be a $\left\{P_{2}, P_{3}\right\}$-factor of $K$ granted by Corollary 6. Consider a $P_{3}(a, b, c)$ of $F$. Let $\gamma(a)=\partial R_{(a, b, c)} \cap \partial R_{a}$ and $\gamma(c)=\partial R_{(a, b, c)} \cap \partial R_{c}$ (i.e., two connected polygonal curves). Denote the two endpoints of $\gamma(a)$ and $\gamma(c)$ by $p_{1}, p_{2}$ and $q_{1}, q_{2}$, respectively, in clockwise order along $\partial R_{(a, b, c)}$. Let $\kappa(a)$ be the polygonal path along $\partial R_{(a, b, c)}$ from $p_{1}$ to $q_{1}$, and let $\kappa(c)$ be the polygonal path along $\partial R_{(a, b, c)}$ from $q_{1}$ to $p_{1}$ in clockwise order. As a result, $\partial R_{(a, b, c)}=\kappa(a) \cup \kappa(c), \partial R_{a} \cap \partial R_{(a, b, c)} \subset \kappa(a)$, and $\partial R_{c} \cap \partial R_{(a, b, c)} \subset \kappa(c)$ (Fig. 10).

We define a graph $J_{F}$. Let the node set $V\left(J_{F}\right)$ be the set of all two extremal nodes from every $P_{3} \in F$. Two nodes $u, v \in V\left(J_{F}\right)$ are connected by an edge if and only if at least one of the following conditions is satisfied (see Fig. 11):

- $u$ and $v$ are in the same $P_{3}$ of $F$,

- $\kappa(u) \cap \kappa(v)$ contains infinitely many points (this includes the case where the cells $R_{u}$ and $R_{v}$ have common boundary points),

- $R_{u}$ and $R_{v}$ lie at the opposite side of two bad points of a bad $P_{2}$ in $F$.

Observe that $J_{F}$ is planar: We can draw $J_{F}$ as follows. Place a point $p(a) \operatorname{in} \operatorname{int}\left(R_{a}\right)$ for every $a \in V\left(J_{F}\right)$, and connect the points corresponding to adjacent nodes of $J_{F}$ by curves without crossings: For a $P_{3}(a, b, c)$ of $F$, the two points, $p(a)$ and $p(c)$, can be connected by a curve within $R_{(a, b, c)}$. If $\kappa\left(u_{1}\right) \cap \kappa\left(v_{1}\right)$ contains a segment, then $p\left(u_{1}\right)$
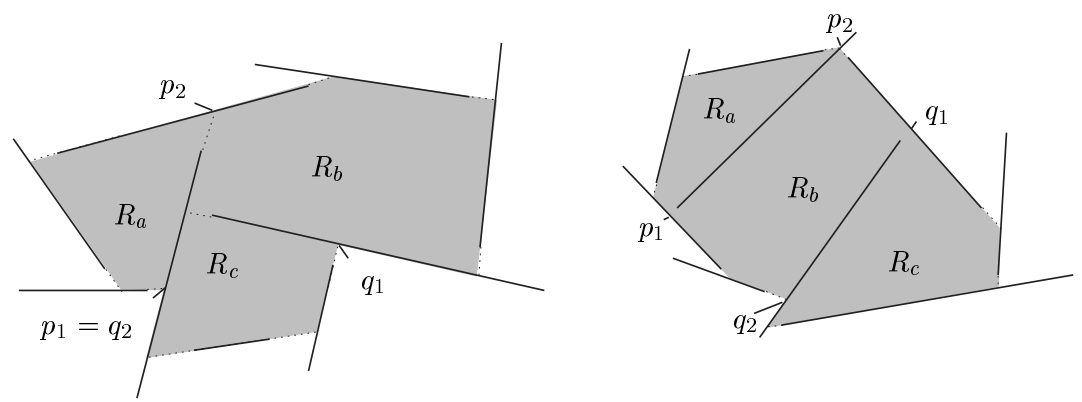

Fig. 10. Denote the two endpoints of $\alpha=\partial R_{(a, b, c)} \cap \partial R_{a}$ and $\gamma=\partial R_{(a, b, c)} \cap \partial R_{c}$ by $p_{1}, p_{2}$ and $q_{1}, q_{2}$, respectively. 


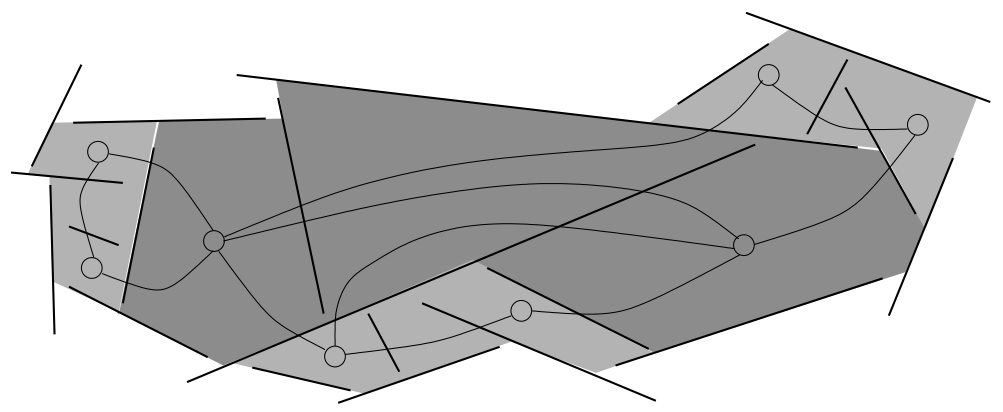

Fig. 11. Edges of graph $J_{F}$ defined on the extremal nodes of $P_{3}$ 's of $F$.

and $p\left(v_{1}\right)$ can be connected to this segment within $R_{\left(u_{1}, u_{2}, u_{3}\right)}$ and $R_{\left(v_{1}, v_{2}, v_{3}\right)}$, respectively, where $\left(u_{1}, u_{2}, u_{3}\right) \in F$ and $\left(v_{1}, v_{2}, v_{3}\right) \in F$. Finally, if $R_{u}$ and $R_{v}$ lie at the opposite sides of the bad points $\tau\left(e_{1}\right)$ and $\tau\left(e_{2}\right)$ of a bad $P_{2} a b$ of $F$, then $p(u)$ and $p(v)$ can be connected to $\tau\left(e_{1}\right)$ and $\tau\left(e_{2}\right)$ within $R_{u}$ and $R_{v}$, respectively; and $\tau\left(e_{1}\right)$ and $\tau\left(e_{2}\right)$ can be connected in $R_{a b}$.

According to the four color theorem [2], [3], $J_{F}$ has an independent set $I_{F} \subset V\left(J_{F}\right)$ of size $\left|I_{F}\right| \geq\left\lceil\left|V\left(J_{F}\right)\right| / 4\right\rceil$. Putting $T_{F}=V\left(J_{F}\right) \backslash I_{F}$, we have $\left|T_{F}\right| \leq\left\lfloor 3\left|V\left(J_{F}\right)\right| / 4\right\rfloor$.

$T_{F}$ has one or two nodes in every $P_{3}$ of $F$. Indeed, $I_{F}$ cannot contain both nodes from a $P_{3}$ of $F$, because they are connected by an edge in $J_{F}$. Hence, at least half of the $P_{3}$ 's of $F$ contain only one node of $T_{F}$, and at most half of them contain two.

We create a new decomposition $\hat{F}$ of $V(K)$ by splitting some of the $P_{3}$ 's of $F$ into a $P_{2}$ and a single node: If $(a, b, c)$ is a $P_{3}$ of $F$ and $c \in I_{F}$ (therefore $\left.a \in T_{F}\right)$, then we split $(a, b, c)$ into $(a, b)$ and $c$. All other $P_{3}$ 's and all $P_{2}$ 's of $F$ are put into $\hat{F}$ intact. Let $q_{1}, q_{2}$, and $q_{3}$ be the number of single nodes, $P_{2}$ 's, and $P_{3}$ 's in $\hat{F}$, respectively. Clearly, $q_{1}+2 q_{2}+3 q_{3}=n+1$. Every single node is obtained from a $P_{3}$ of $F$ with one node in $I_{F}$, hence $q_{3} \leq q_{1}$.

Now we define a set of light sources $S(\hat{F})$. Place two light sources into each $P_{3}$ of $\hat{F}$ as described in Lemma 17. Place one light source into each $P_{2}$ of $\hat{F}$ as described in Lemma 19. (The proof of Lemma 19 suggests four possibilities for the location of a light source in a bad pair. We use the freedom of choice in the proof of Lemma 21.) There are no light sources in the single nodes of $\hat{F}$. All in all, we used at most $\lfloor(n+1) / 2\rfloor$ light sources.

Lemma 21. The light sources of $S(\hat{F})$ can be arranged so that they collectively illuminate every point of $\bigcup L$.

Proof. We prove that every portion of $\bigcup L$ on the boundary of every convex cell of the convex partition is illuminated by some light source of $S(\hat{F})$.

The portions of $\bigcup L$ in and on the boundaries of $R_{(a, b, c)}$, where $(a, b, c)$ is a $P_{3}$ of $\hat{F}$, are illuminated according to Lemma 17(i). Likewise the portions in and on the boundary of $R_{a b}$, where $a b$ is a good $P_{2}$ of $\hat{F}$. If $a b$ is a bad pair of $\hat{F}$, then one of four arbitrarily short segments in the neighborhood of one of its bad points is not illuminated from the side of $R_{a b}$. The boundary of $R_{a}$ where $a \in I_{F}$ is not illuminated from the side of $R_{a}$. 


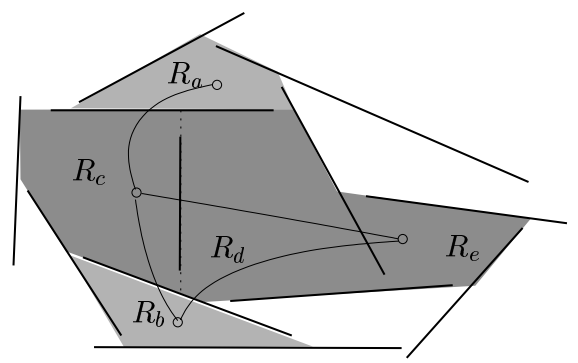

Fig. 12. A bad $P_{2},(c, d)$, is part of the $P_{3}(c, d, e)$.

We need to show that the boundaries of cells corresponding to single nodes of $\hat{F}$ and the short segments at bad points of bad pairs of $\hat{F}$ are illuminated from the opposite side. There are three cases to consider:

(1) If $a$ and $b$ are single nodes of $\hat{F}$, then $a$ and $b$ are vertices of the graph $J_{F}$, and $a, b \in I_{F}$. The cells $R_{a}$ and $R_{b}$ cannot have more than one common boundary points, because then $J_{F}$ has an edge between $a$ and $b$.

(2) If $a b$ and $b c$ are two bad pairs of $\hat{F}$ and $R_{a b}$ and $R_{c d}$ have a common boundary, then the light sources $s_{a b} \in S(\hat{F})$ and $s_{c d} \in S(\hat{F})$ can always be arranged so that every point in $\partial R_{a b} \cap \partial R_{c d}$ is illuminated by at least one of them.

(3) Suppose that $a$ and $b$ are single nodes (so $a, b \in I_{F}$ ) and $c d$ is a bad $P_{2}$ of $\hat{F}$, furthermore, $R_{a}$ and $R_{b}$ are located at the other side of the bad points of $c d$. (See Fig. 12.)

$c d$ cannot be a bad $P_{2}$ of $F$, because then the nodes $a$ and $b$ are connected by an edge in the graph $J_{F}$, therefore $a$ and $b$ cannot be in $I_{F}$. Therefore, $c d$ was obtained by splitting a $P_{3}(c, d, e)$ of $F$ into a pair $c d$ and a singleton $e$. This implies that $e$ is also a node of the graph $J_{F}$ and $e \in I_{F}$.

Now observe that the two bad points of $c d$ are also the two endpoints of the curve $\gamma(c)$ defined for $(c, d, e)$. Since $R_{a}$ and $R_{b}$ are at the other side of the two bad points of $c d$, either $\partial R_{a}$ or $\partial R_{b}$ contains a common segment with $\kappa(e)$. Therefore, $J_{F}$ has an edge between $e$ and, say, $b$, so they cannot be in an independent set of $J_{F}$, a contradiction.

\section{Acknowledgments}

I am indebted to G.Y. Katona for pointing out Kaneko's theorem, and to Emo Welzl and Günter Rote for many useful comments and suggestions.

\section{References}

1. J. Akiyama, D. Avis, and H. Era, On a \{1, 2\}-factor of a graph, TRU Math. 16 (1980), 97-102.

2. K. Appel and W. Haken, Every planar map is four colorable, part i, discharging, Illinois J. Math. 21 (1977), 429-490.

3. K. Appel, W. Haken, and J. Koch, Every planar map is four colorable, part ii, reducibility, Illinois J. Math. 21 (1977), 491-567. 
4. F. Berman, D. Johnson, T. Leighton, P. W. Shor, and L. Snyder, Generalized planar matching, J. Algorithms 11 (1990), 153-184.

5. J. Czyzowicz, I. Rival, and J. Urrutia, Galleries, light matchings and visibility graphs, in: Proc. Algorithms and Data Structures (Ottawa, ON, 1989), vol. 382 of Lecture Notes in Computer Science, Springer-Verlag, Berlin, 1989, pp. 316-324.

6. J. Czyzowicz, E. Rivera-Campo, and J. Urrutia, Illuminating rectangles and triangles on the plane, J. Combin. Theory Ser. B 57 (1993), 1-17.

7. J. Czyzowicz, E. Rivera-Campo, J. Urrutia, and J. Zaks, On illuminating line segments in the plane, Discrete Math. 137 (1995), 147-153.

8. L. Fejes Tóth, Illumination of convex disks, Acta Math. Acad. Sci. Hungar. 29 (1977), 355-360.

9. H. Hadwiger, Ungelöste Probleme - nr. 38, Elem. Math. 15 (1960), 130-131.

10. D. Hartvigsen and P. Hell, The $k$-piece packing problem, presented at the 17 th International Symposium on Mathematical Programming (Atlanta, GA), 2000.

11. F. Hoffmann, On the rectilinear art gallery problem, in: Proc. 17th Internat. Coll. Automata, Lang. and Prog. (Warwick, 1990), vol. 443 of Lecture Notes in Computer Science, Springer-Verlag, Berlin, 1990, pp. $717-728$.

12. G. Jennings and W. Lenhart, An art gallery theorem for line segments in the plane, Pattern Recognition Lett. 14 (1993), 727-732.

13. A. Kaneko, A necessary and sufficient condition for the existence of a path factor every component of which is a path of length at least two, submitted, 2000.

14. M. Kano, G. Katona, and Z. Király, Packing paths of length at least two, submitted, 2001

15. D. G. Kirkpatrick and P. Hell, On the completeness of a generalized matching problem, in: Proc. 10th ACM Symp. on Theory of Computing (San Diego, CA, 1978), ACM Press, New York, 1978, pp. 240-245.

16. H. Martini and V. Soltan, Combinatorial problems on the illumination of convex bodies, Aequationes Math. 57 (3) (1999), 121-152.

17. J. O'Rourke, Art Gallery Theorems and Algorithms, Oxford University Press, New York, 1987.

18. Cs. D. Tóth, Illuminating both sides of line segments, in: Discrete and Computational Geometry (J. Akiyama, M. Kano, and M. Urabe, eds.), vol. 2098 of Lecture Notes in Computer Science, SpringerVerlag, Berlin, 2001, pp. 370-380.

19. Cs. D. Tóth, Illumination in the presence of opaque line segments in the plane, Comput. Geom. Theory Appl. 21 (2002), 193-204.

20. J. Urrutia, Art gallery and illumination problems, in: Handbook on Computational Geometry, J. R. Sack and J. Urrutia, eds., Elsevier, Amsterdam, 2000, pp. 973-1027.

21. F. A. Valentine, Visible shorelines, Amer. Math. Monthly 77 (1970), 146-152.

22. J. Zaks, A note on illuminating line segments in the plane, manuscript, 1993.

Received December 18, 2000, and in revised form December 26, 2002. Online publication August 6, 2003. 